\title{
Effects of rapid thermal annealing on device characteristics of InGaAs/GaAs quantum dot infrared photodetectors
}

\author{
L. Fu, ${ }^{\text {a) }}$ H. H. Tan, I. McKerracher, J. Wong-Leung, and C. Jagadish \\ Department of Electronic Materials Engineering, Research School of Physical Sciences and Engineering, \\ The Australian National University, Canberra, Australian Capital Territory 0200, Australia
}

N. Vukmirović and P. Harrison
School of Electronic and Electrical Engineering, University of Leeds, Leeds LS2 9JT, United Kingdom

(Received 7 November 2005; accepted 17 March 2006; published online 13 June 2006)

In this work, rapid thermal annealing was performed on InGaAs/GaAs quantum dot infrared photodetectors (QDIPs) at different temperatures. The photoluminescence showed a blueshifted spectrum in comparison with the as-grown sample when the annealing temperature was higher than $700{ }^{\circ} \mathrm{C}$, as a result of thermal interdiffusion of the quantum dots (QDs). Correspondingly, the spectral response from the annealed QDIP exhibited a redshift. At the higher annealing temperature of $800{ }^{\circ} \mathrm{C}$, in addition to the largely redshifted photoresponse peak of $7.4 \mu \mathrm{m}$ (compared with the $6.1 \mu \mathrm{m}$ of the as-grown QDIP), a high energy peak at $5.6 \mu \mathrm{m}(220 \mathrm{meV})$ was also observed, leading to a broad spectrum linewidth of $40 \%$. This is due to the large interdiffusion effect which could greatly vary the composition of the QDs and thus increase the relative optical absorption intensity at higher energy. The other important detector characteristics such as dark current, peak responsivity, and detectivity were also measured. It was found that the overall device performance was not affected by low annealing temperature, however, for high annealing temperature, some degradation in device detectivity (but not responsivity) was observed. This is a consequence of increased dark current due to defect formation and increased ground state energy. (C) 2006 American Institute of Physics. [DOI: 10.1063/1.2202704]

\section{INTRODUCTION}

With the rapid progress in the growth of self-assembled quantum dots (QDs), their application to high performance optoelectronic devices, such as QD laser diodes and QD infrared photodetectors, have been successfully demonstrated. ${ }^{1-3}$ For developing future optical communication networks, monolithic integration is a key issue in order to achieve better optical performance, compact size, and low losses. One conventional way of fabricating photonic integrated circuits (PICs) involves repeated etching and regrowth processes, which place a high constraint on the etching and epitaxial growth techniques. In comparison, the approach of postgrowth intermixing (interdiffusion) developed in recent years has offered simple and more practicable schemes for PICs through the modification of the potential profile and thus the optical properties of the quantum-confined heterostructures. For example, quantum well intermixing (QWI) has been successfully employed to tune the wavelength of quantum well $(\mathrm{QW})$ lasers ${ }^{4,5}$ and $\mathrm{QW}$ infrared photodetectors (QWIPs), ${ }^{6,7}$ as well as in the fabrication of integrated devices. ${ }^{8}$ However, due to the large surface area and strain effect, interdiffusion behavior in QDs is much more complicated than in QWs. Hence, the technologies that have been developed in QWs cannot be transferred directly to QDs. Although interdiffusion studies have been carried out on

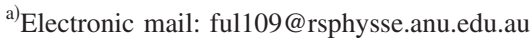

various QD structures using the methods of rapid thermal annealing (RTA), ${ }^{9,10}$ ion implantation, ${ }^{11,12}$ and dielectric capping, ${ }^{12,13}$ few device results have emerged. Midinfrared $(3-5 \mu \mathrm{m})$ and far-infrared $(8-14 \mu \mathrm{m})$ photodetectors ${ }^{3,14,15}$ based on QDs have been predicted to have the advantages of normal incidence and high temperature operation, larger responsivity, and detectivity, in comparison to their QW counterpart. However, the operating wavelength of quantum dot infrared photodetectors (QDIPs) is difficult to predict and control accurately due to the extremely sensitive selforganized process of the dot formation. Structure designs such as dots in a well ${ }^{15}$ have been proposed and demonstrated with flexibilities in altering the operating wavelength of QDIPs by varying the thickness of the quantum well surrounding the QDs. However, it still requires highly accurate and reproducible growth conditions. Using the interdiffusion approach, Hwang et al. ${ }^{16}$ reported the detection wavelength tuning of an InGaAs/GaAs QDIP after thermal treatment at $700{ }^{\circ} \mathrm{C}$ for 1 min under $\mathrm{SiO}_{2}$ capping. A $22 \mathrm{meV}$ redshift of photocurrent peak was obtained but with a large degradation in device responsivity (detectivity not reported). In this work, a more extensive investigation was carried out to study the postgrowth annealing effects on the intersubband transition of a 15-layer InGaAs/GaAs QDIP. The device characteristics including spectral response, dark current, responsivity, and detectivity were fully investigated and compared after a series of annealing conditions and the implications for the future development of multicolor and broadband photodetectors are also discussed. 


\section{EXPERIMENT}

The QDIP structure used in this work was a $n-i-n$ structure $^{17}$ grown on a semi-insulating GaAs (001) substrate by metal-organic chemical vapor deposition (MOCVD). Arsine $\left(\mathrm{AsH}_{3}\right)$, trimethylgallium (TMGa), and trimethylindium (TMIn) were used as sources for arsenic, gallium, and indium, respectively. The structure contained 15 layers of 5.7 ML (monolayer) undoped InGaAs (nominally 50\%) QDs, separated by $50 \mathrm{~nm}$ of GaAs barrier layers and sandwiched between two highly Si-doped top $(300 \mathrm{~nm})$ and bottom $(1000 \mathrm{~nm}) \mathrm{GaAs}$ contact layers. The growth details have been published in Ref. 18. Rapid thermal annealing was performed on the samples at temperatures ranging from 650 to $800{ }^{\circ} \mathrm{C}$ for $30 \mathrm{~s}$ under Ar flow. During annealing, the samples were sandwiched between two fresh pieces of GaAs to minimize desorption of As from the surface. Before device fabrication, the samples were examined by cross-sectional transmission electron microscopy (XTEM) using a Philips CM 300 microscope and photoluminescence (PL) using a diode-pumped solid-state frequency-doubled green laser at $532 \mathrm{~nm}$ through a $0.5 \mathrm{~m}$ monochromator and a cooled InGaAs photodetector. Then, all of the samples were fabricated into $250 \times 250 \mu \mathrm{m}^{2}$ mesa structures using standard photolithography, wet chemical etching, and metallization processes. The 150- $\mu \mathrm{m}$-diameter round top contact and the bottom contact were formed by electron-beam evaporation of $\mathrm{Ge} / \mathrm{Ni} / \mathrm{Au}$ and alloying at $380{ }^{\circ} \mathrm{C}$ for $60 \mathrm{~s}$. The devices were then mounted in a liquid nitrogen cooled Dewar for further characterization.

\section{RESULTS}

\section{A. Cross-sectional transmission electron microscopy and photoluminescence}

Figure 1(a) shows the TEM micrographs of the as-grown 15-layer InGaAs/GaAs QDIPs used in the study. With all 15 QD layers displayed in the structure, no obvious formation of lattice defects such as dislocations can be observed, suggesting that good material quality has been maintained after 15-layer dot growth. Figure 1(a) also shows the high resolution (HR) TEM image of a typical QD with a thin wetting layer clearly observed underneath the QD. The QD has a truncated cone shape with $\sim 5 \mathrm{~nm}$ height and $\sim 20 \mathrm{~nm}$ base diameter, which agrees well with our atomic force microscopy measurement. ${ }^{18}$ The HR image also exhibits some contrast between the middle and outside of the QD, which could have resulted from the nonuniform strain field and/or In distribution in the QDs. The room temperature PL also confirmed the good quality of the QDIP structure, as shown in Fig. 1(b). The main peak of the spectrum was from the dot luminescence and the high energy peak $(\sim 867 \mathrm{~nm})$ is from the GaAs capping layer/substrate. In addition, a small shoulder to the main QD peak arising from the wetting layer can also be noted. The high intensity QD peak is located at $1140 \mathrm{~nm}$ with the full width at half maximum (FWHM) of $80 \mathrm{meV}$.

Figure 2 shows the $77 \mathrm{~K}$ PL spectra taken from the samples which were annealed from 650 to $800{ }^{\circ} \mathrm{C}$ for $30 \mathrm{~s}$. The QD peak position from the sample annealed at $650^{\circ} \mathrm{C}$
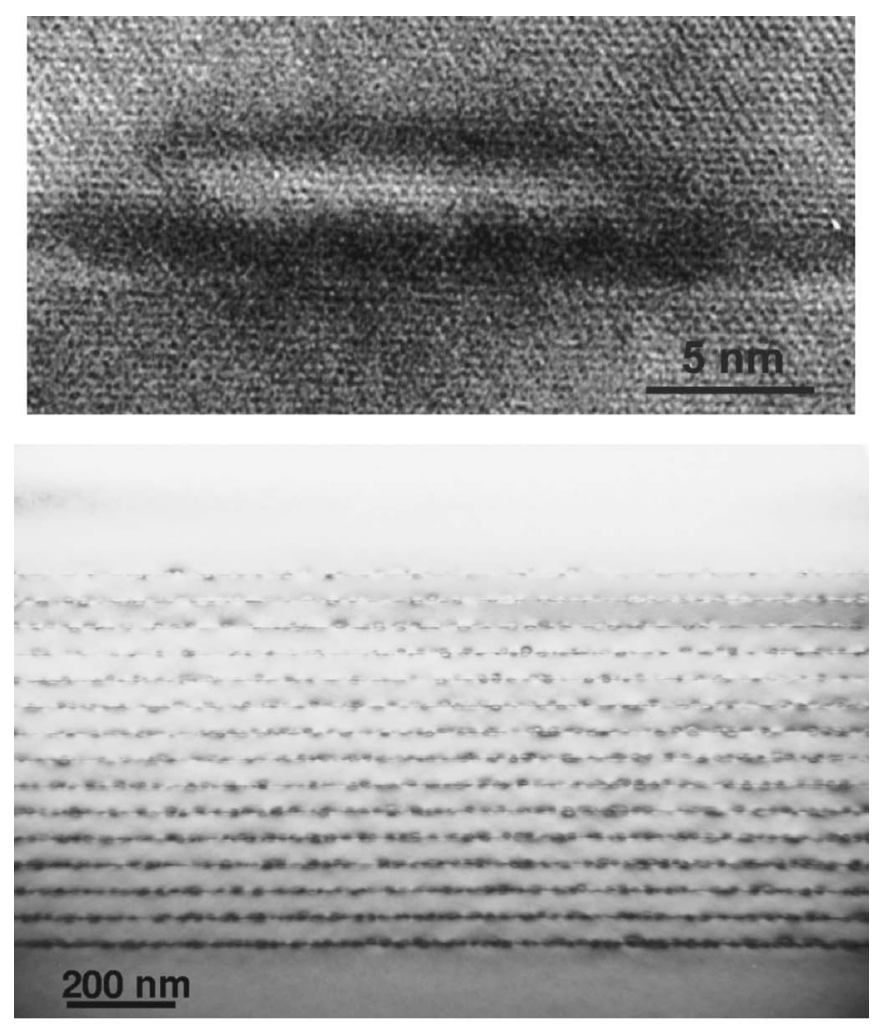

(a)

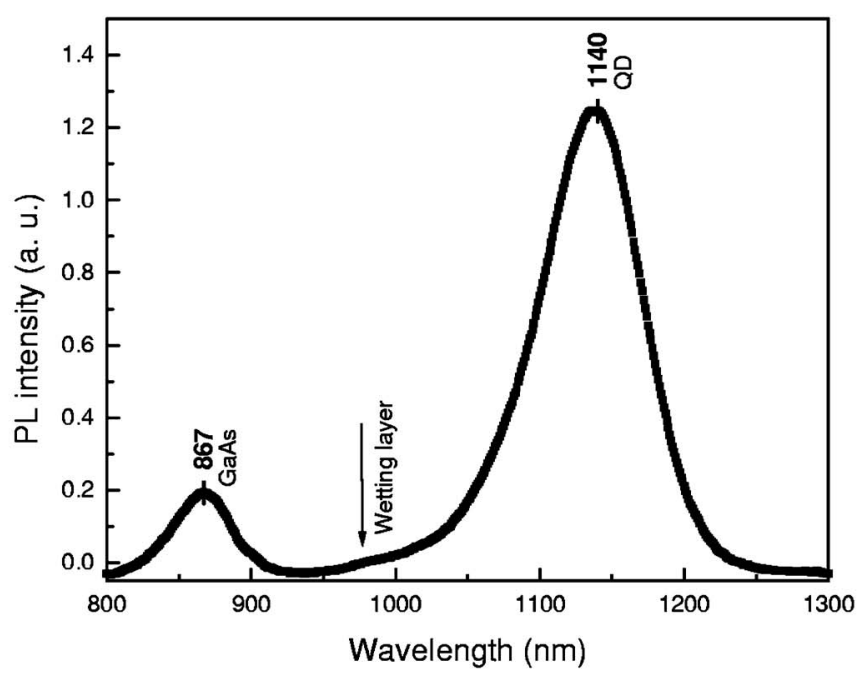

(b)

FIG. 1. (a) Bottom: XTEM image of the as-grown 15-layer QDIP structure; top: High resolution XTEM image of a single QD within the 15-layer QDIP structure. (b) Room temperature PL from the as-grown 15-layer QDIP structure.

does not show any change compared with that from the as-grown sample. Then, with increasing of the annealing temperature to $700{ }^{\circ} \mathrm{C}$, the spectrum is blueshifted with a narrowing of the linewidth. The PL energy shift as well as the ratio of the FWHM between the annealed and as-grown samples as a function of annealing temperature are shown in the inset of Fig. 2. It can be seen clearly that with increasing annealing temperature, more intermixing was created, which is consistent with an increase of PL blueshift and a reduction of linewidth. This has been observed previously in 


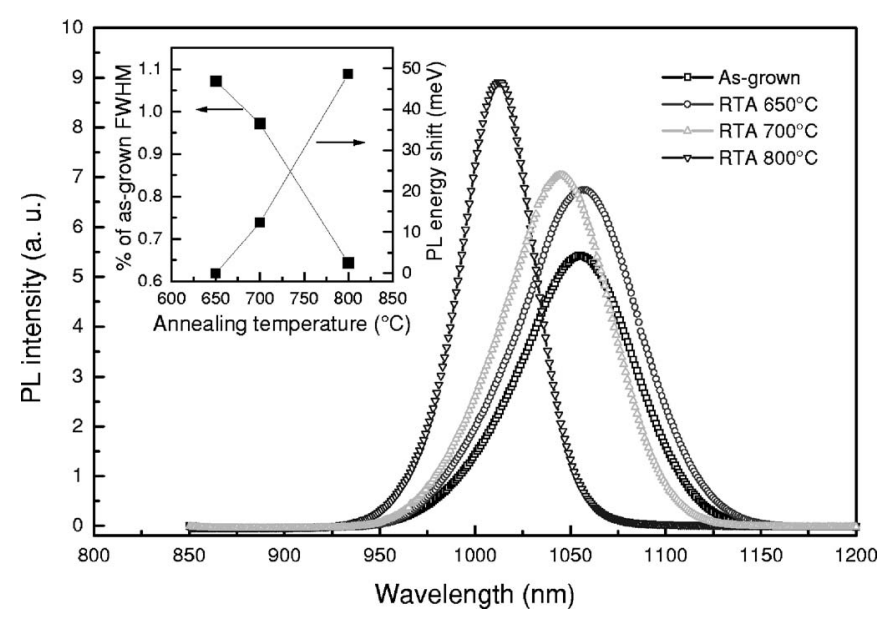

FIG. 2. The $77 \mathrm{~K}$ PL spectra for the QDIP samples annealed at different temperatures. Inset shows the PL energy shifts (right) and the linewidth (left) as a function of annealing temperature.

many studies, as a result of significant thermal interdiffusion. ${ }^{9,10,13,19}$ However, for our 15-layer QDIP structure, the interdiffusion was initiated at a lower temperature of $700{ }^{\circ} \mathrm{C}$ [compared with $750{ }^{\circ} \mathrm{C}$ for single and 3-layer QDs (Ref. 10)], due to a larger accumulated strain. In addition, Fig. 2 also exhibits an enhanced QD luminescence with annealing temperature. This is mainly attributed to the spectral narrowing due to the improved QD size homogeneity after interdiffusion as well as the reduction of grown-in defect density around QDs. ${ }^{9,13}$

\section{B. Detector spectral response}

The normal incidence spectral response was measured with a Nicolet Impact 400 Fourier transform infrared (FTIR) spectrometer and a SRS 570 low-noise current preamplifier. Figure 3 shows the $77 \mathrm{~K}$ spectral response from the device fabricated from the as-grown sample at various positive biases (on the top contact layer with respect to the bottom contact layer). At lower biases, the spectra are very broad and clearly consist of a short wavelength peak at $4.5 \mu \mathrm{m}$ $(274 \mathrm{meV})$ and a long wavelength peak at $6.1 \mu \mathrm{m}$

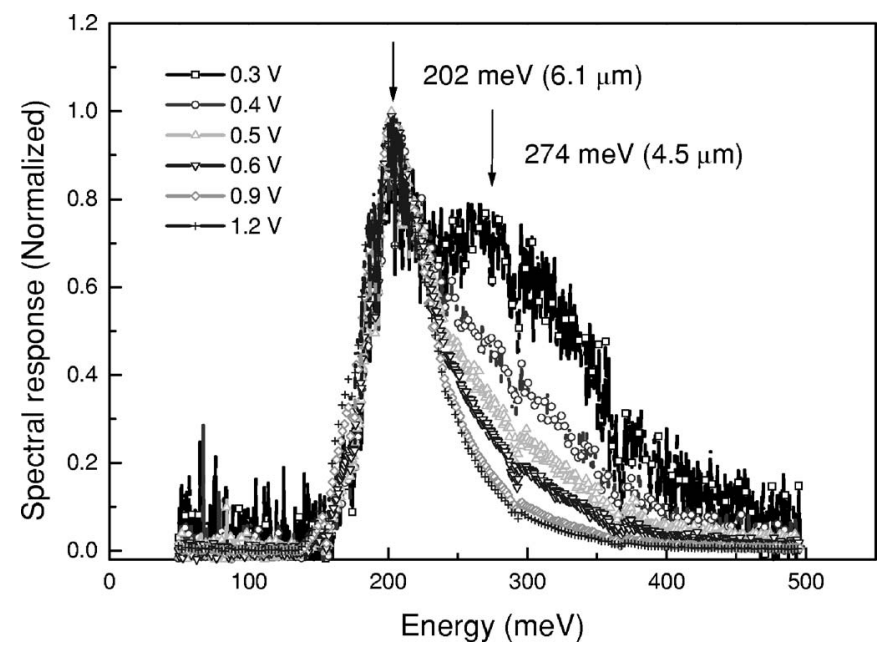

FIG. 3. The spectral response from the as-grown QDIP at various external biases at $77 \mathrm{~K}$.

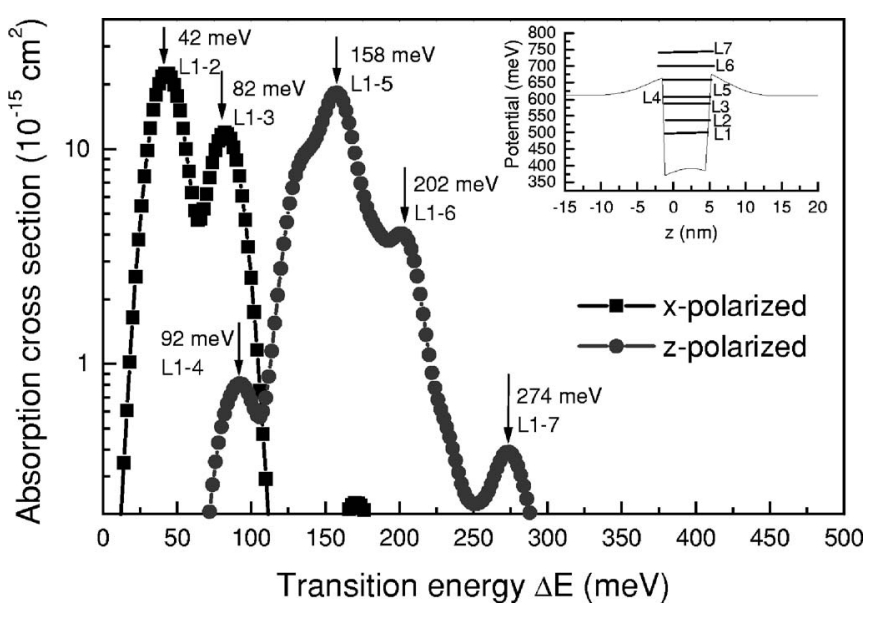

FIG. 4. The calculated spectrum of optical absorption cross sections for $65 \%$ InGaAs/GaAs QDs. Inset shows the schematic of the conduction band diagram and relevant energy levels.

(202 meV). With an increase of the bias, the intensity of the $4.5 \mu \mathrm{m}$ peak reduces rapidly and the $6.1 \mu \mathrm{m}$ peak becomes the dominant peak. To clarify the origin of the two peaks, the spectrum of optical absorption from the ground state was calculated theoretically. Dots in the shape of truncated cones with a base diameter of $20 \mathrm{~nm}$ and a height of $5 \mathrm{~nm}$ were assumed in accordance with the HR TEM results in Fig. 1(a). A wetting layer width of $4 \mathrm{ML}$ and In content of 0.65 (larger than the nominal value for growth) were assumed. The energy levels and wave functions were calculated using the eight-band $k \cdot p$ method with the strain distribution taken into account via the continuum mechanical model. ${ }^{20}$ In the calculation the dot was embedded in a large cylinder, so that both the wetting layer and the continuum states were discretized that way. The optical absorption cross section on the transition between ground state and any excited state (bound, wetting layer, or continuum) was calculated as in Ref. 21. According to the calculation, the largest optical absorption cross section for $x$-polarized radiation (in-plane direction) and $z$-polarized radiation (growth direction) occurs at $42 \mathrm{meV}$ (L1-2) and $158 \mathrm{meV}$ (L1-5), respectively, as displayed in Fig. 4. As the states of these energies are below the effective potential barrier (see the schematic of the dot potential in the inset of Fig. 4) they are localized (or partially localized) to the dot which did not result in detectable photocurrent because the electrons from them have a low escape probability. ${ }^{22}$ Therefore we did not observe any peak related to this transition experimentally. The largest optical absorption cross section for the bound-to-continuum $(B-C)$ transition under $z$-polarized light occurs at $202 \mathrm{meV}$ (L1-6) and the next significant transition is at $274 \mathrm{meV}$ (L1-7, 10\% of the former). These two peaks correspond very well with the results from the spectral response measurements. At low biases, although more carriers were excited to the lower energy continuum state (L6), many of them could not contribute to photocurrent due to the small electric field. On the other hand, the carriers excited to the higher energy state (L7) travel more easily since they are less likely to be trapped back to the QD energy potentials, thus forming the $4.5 \mu \mathrm{m}$ peak $(274 \mathrm{meV})$ as shown clearly at the bias of $0.3 \mathrm{~V}$ in Fig. 


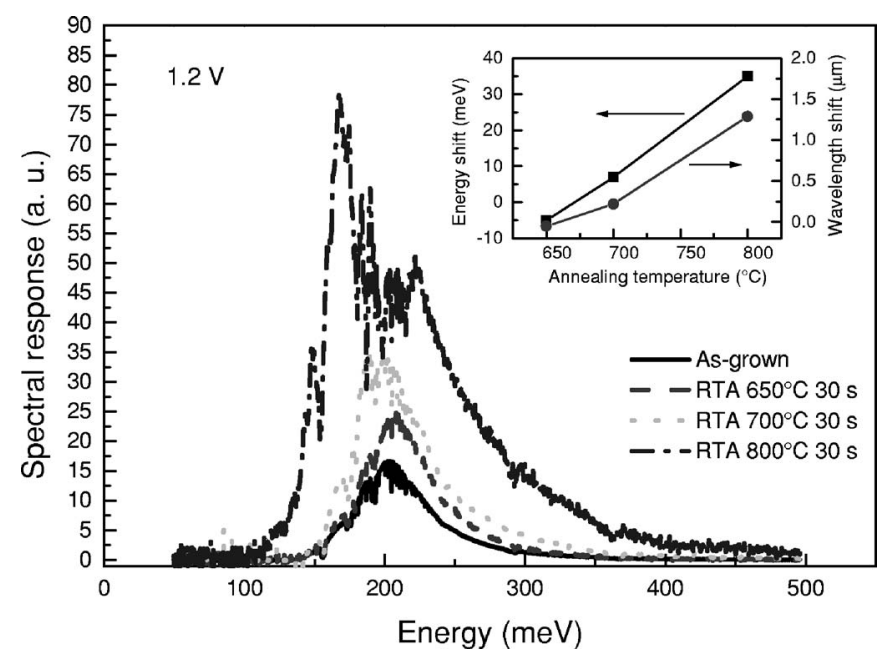

FIG. 5. The spectral responses at $1.2 \mathrm{~V}$ from the QDIPs which were fabricated from samples annealed at different temperatures. Inset shows the device intersubband transition wavelength shift (right) as well as the energy shift (left) as a function of annealing temperature for annealed QDIPs compared with the as-grown QDIP.

3. With an increase of the bias, the carrier collection efficiency is improved much more and most of the carriers created due to the $202 \mathrm{meV}$ transition can be swept towards the collector, contributing dominantly to the photocurrent at $6.1 \mu \mathrm{m}$.

Figure 5 shows the spectral responses from the QDIPs annealed at different temperatures. The spectra exhibit redshifted peak wavelengths for the annealing temperatures higher than $650{ }^{\circ} \mathrm{C}$. The energy and wavelength shifts between the annealed devices and as-grown device are plotted in the inset of Fig. 5. The wavelength redshifting for annealed QDIPs is a result of thermal interdiffusion. It is well known $^{23}$ that interdiffusion of quantum-confined structures (QWs and QDs) is able to modify the shape of their potential profiles and thus cause an increase in the interband transition energy (blueshifted PL) and a decrease in that of the intersubband transition (redshifted intersubband photocurrent). Since the intersubband photocurrent only involves the transitions within conduction band, the energy redshifts obtained in the inset of Fig. 5 are smaller than PL blueshifts in the inset of Fig. 2.

It is interesting to note that for the $800{ }^{\circ} \mathrm{C}$ annealed QDIP, in addition to the large $35 \mathrm{meV}$ energy shift (or $1.3 \mu \mathrm{m}$ wavelength shift) obtained from its main peak at $167 \mathrm{meV}(7.39 \mu \mathrm{m})$ [compared with the $210 \mathrm{meV}(6.1 \mu \mathrm{m})$ peak of the as-grown sample], a second peak at higher energy $(\sim 220 \mathrm{meV})$ is clearly displayed in the spectrum, which has not been observed for the other samples. In fact, this high energy peak was detected at all bias conditions from the $800{ }^{\circ} \mathrm{C}$ annealed QDIP and its relative intensity to the low energy $167 \mathrm{meV}$ peak also changed with the bias, as shown in Fig. 6(a). Since the $800{ }^{\circ} \mathrm{C}$ RTA introduced the largest degree of interdiffusion, one of its direct effects was the outdiffusion of In from the QD to its surrounding barriers, leading to a reduction in the QD In composition and thus a redistribution of the QD energy states and their optical absorption strength. We have approximately modeled these

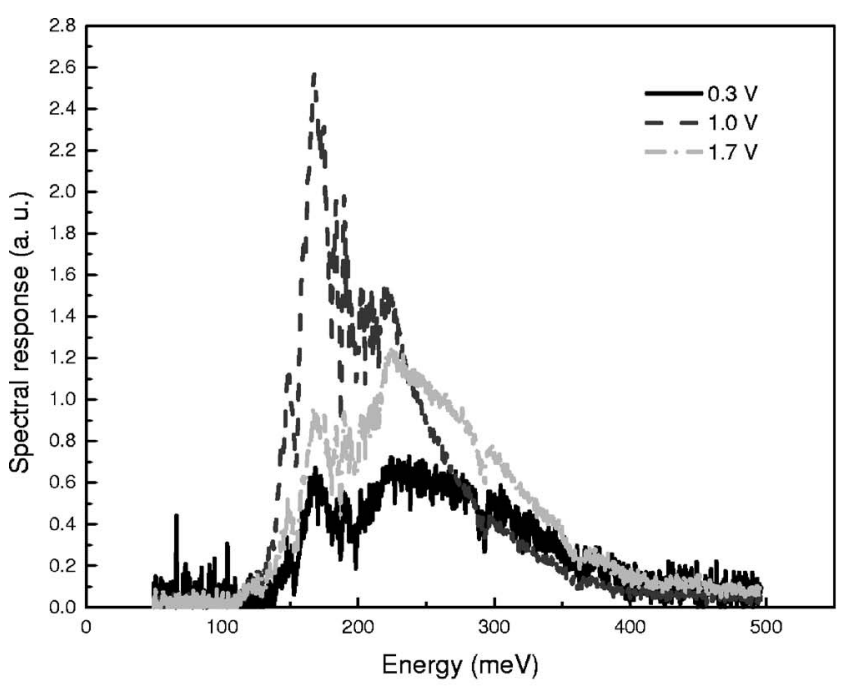

(a)

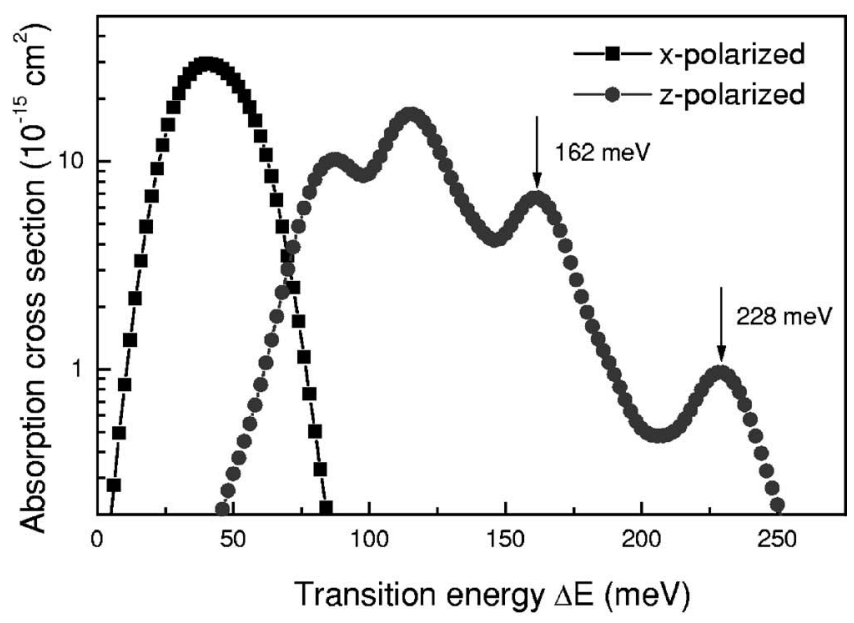

(b)

FIG. 6. (a) The spectral responses from the $800{ }^{\circ} \mathrm{C}$ annealed QDIP at various positive biases. (b) The calculated spectrum of optical absorption cross sections for $50 \%$ InGaAs/GaAs QDs.

effects by assuming a smaller In content in the dot of 50\%. The calculated absorption spectrum is shown in Fig. 6(b), where the two peaks at continuum states (162 and $228 \mathrm{meV}$ ) show very similar positions to those from the spectral response of our $800{ }^{\circ} \mathrm{C}$ annealed QDIP. Note that the relative absorption cross section for the high energy peak is increased to $15 \%$ of the low energy peak (10\% for the $65 \%$ InGaAs QDs), which may be a reason for the detection of the high energy peak at all biases. Nevertheless, this is just a simplification of the complicated interdiffusion process. More detailed modeling is needed to simulate the intersubband transitions in interdiffused QDs accurately. Due to the presence of two peaks, the $800{ }^{\circ} \mathrm{C}$ annealed QDIP has a broad linewidth $(\Delta \lambda / \lambda \sim 40 \%)$, exhibiting a desirable feature for broadband infrared detector applications.

\section{Dark current}

The dark-current-voltage $(I-V)$ characteristics were measured using a Hewlett-Packard 41408 voltage source at temperatures from 77 to $290 \mathrm{~K}$. Figure 7 shows the bias de- 


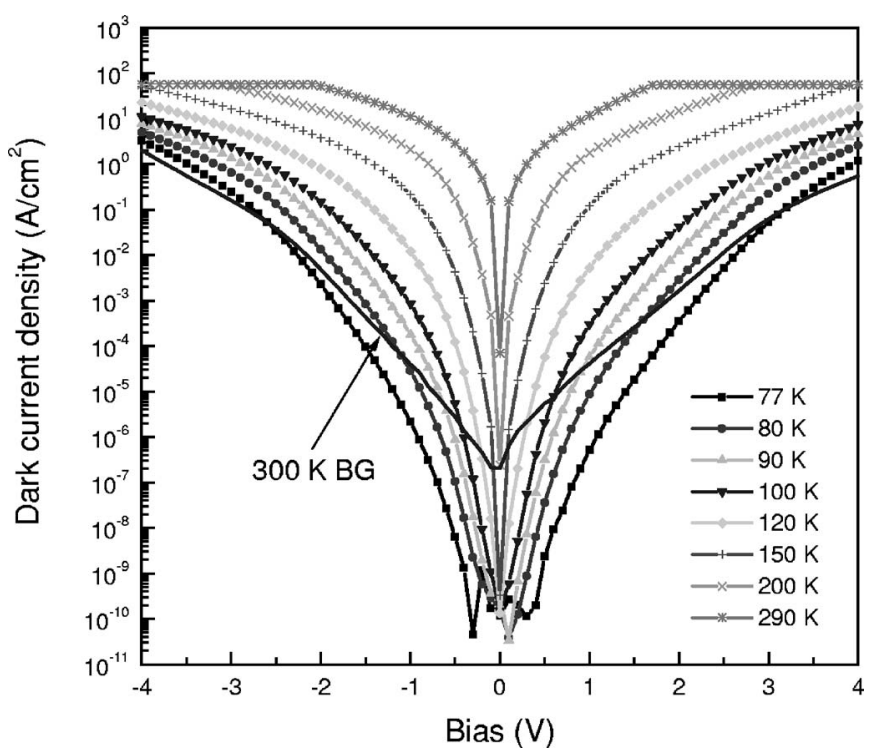

FIG. 7. The $I-V$ characteristics for the as-grown QDIP measured at different temperatures. The $300 \mathrm{~K}$ background photocurrent measured at $77 \mathrm{~K}$ is also shown.

pendent dark current density for the as-grown QDIP at various temperatures. Due to the measurement limit, the dark current at low temperatures and small biases cannot be measured accurately (thus the noisy parts of the curves). Also shown in Fig. 7 is the $300 \mathrm{~K}$ background photocurrent, indicating that the detector is background limited at $77 \mathrm{~K}$ for the wide bias range of $-2.6 \mathrm{~V}<\mathrm{V}_{\text {bias }}<3.2 \mathrm{~V}$. The logarithm of the dark current density versus inverse temperature $(1000 / T)$ at biases from 0.1 to $1.8 \mathrm{~V}$ is shown in Fig. 8. Within the temperature range investigated, each curve shows a consistent increase with the temperature, however, the relationship is not linear. Unlike in the case of QWIPs, where thermionic emission is the major source of dark current when temperature is above $77 \mathrm{~K}$, for QDIPs, it has been proposed ${ }^{24}$ that both field-assisted tunneling and defect-related tunneling (namely, sequential resonant tunneling or defect-assisted tunneling) contribute to the dark current. Consequently, two different regions can be distinguished from the temperature dependent curves. The high temperature region $(>125 \mathrm{~K})$ was ascribed to the thermally limited region (through thermionic emission and field-assisted tunneling), and the low temperature region $(<125 \mathrm{~K})$ to a defect-limited region. From Fig. 8 , this transition behavior can also be identified in all the curves at around $125 \mathrm{~K}$, suggesting that the dark current may arise from different sources. Indeed, since the GaAs barriers of our QDIP structure were grown at a low temperature of $550{ }^{\circ} \mathrm{C}\left(650^{\circ} \mathrm{C}\right.$ is normally used to obtain good quality $\mathrm{GaAs}$ ) to ensure the quality of dot formation, defects are inevitably formed which could result in additional tunneling path for dark current at low temperature. By fitting the linear section of the high temperature region $(>125 \mathrm{~K})$ of the curves using the Arrhenius relation, the activation energy $E_{a}$ is estimated and shows a decrease as a function of bias, as plotted in the inset of Fig. 8.

The $77 \mathrm{~K} I-V$ characteristics for annealed QDIPs are plotted in Fig. 9(a). Annealing causes an increase in the dark current of the devices. Compared with as-grown device, the

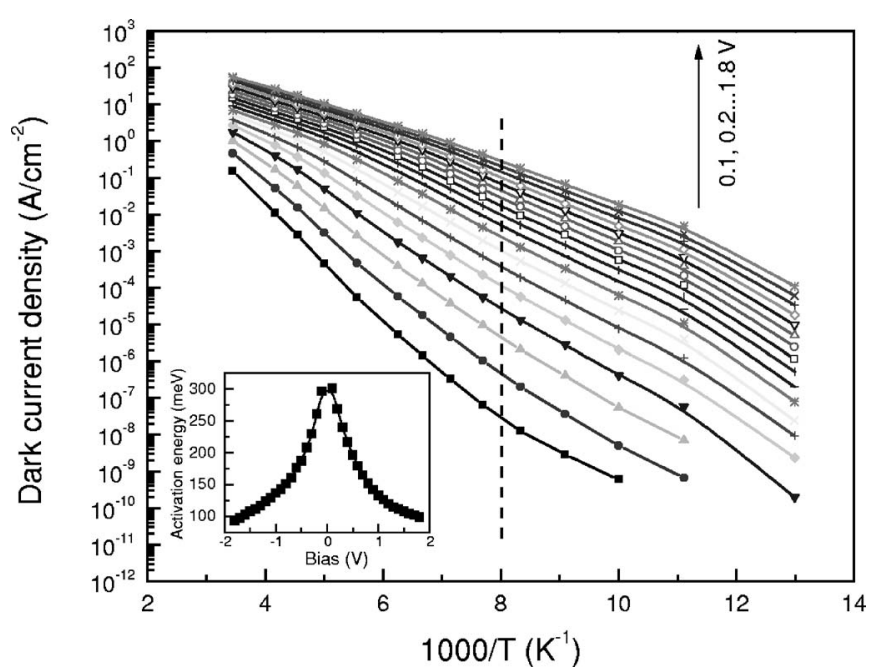

FIG. 8. The dark current density versus inverse temperature $(1000 / T)$ at various biases for the as-grown QDIP. The linear section at the high temperature region $(>125 \mathrm{~K})$ of the curves is used to estimate the dark current activation energy. Inset plots the calculated dark current activation energy as a function of bias.

dark current was increased by one order of magnitude after 650 and $700{ }^{\circ} \mathrm{C}$ annealings whereas $800{ }^{\circ} \mathrm{C}$ annealing increased the dark current by about two orders of magnitude. The temperature dependent dark current density at $0.3 \mathrm{~V}$ for these samples is plotted in Fig. 9(b). The annealed samples display more nonlinear characteristics than the as-grown sample, indicating that their dark currents originated from more temperature-invariant components. We ascribe it to the defects that are generated in the GaAs barriers and at QD interfaces during the high temperature annealing. Normally, annealing is considered to be an effective method to remove defects from the semiconductor structures. However, for a highly strained structure, such as in QDs, it may lead to the formation of extended defects. It has been found by XTEM (Ref. 25) that annealing at $700{ }^{\circ} \mathrm{C}$ of a 30-layer InAs/GaAs QDIP (where some defect centers have already been observed in the as-grown sample) lead to the formation of extended defects due to strain relaxation and dopant-related defect complexes due to high doping in the contact layers. Therefore, up to four orders of magnitude increase of dark current and severely degraded device performance have been observed. For our 15-layer QDIP, although XTEM and PL measurements clearly revealed the good quality of the asgrown QD layers, extended and dopant-related defects may still form during annealing, leading to the increase of dark current. Nevertheless, these defects may be of smaller size and lower concentration in our annealed QDIPs, which have not significantly affected the PL intensity as observed in Fig. 2. High temperature annealing (at $800^{\circ} \mathrm{C}$ ) apparently had much stronger effect on increasing the dark current than the low temperature annealing (at 650 and $700{ }^{\circ} \mathrm{C}$ ). On the other hand, both PL and spectral response results indicate that interdiffusion for our 15-layer QDIPs initiates from $700{ }^{\circ} \mathrm{C}$. It resulted in an increase of ground state energy level and thus a reduction of thermal activation energy of dark current. Therefore, compared with the $650{ }^{\circ} \mathrm{C}$ annealed and asgrown samples, the slope of the dark current density versus 


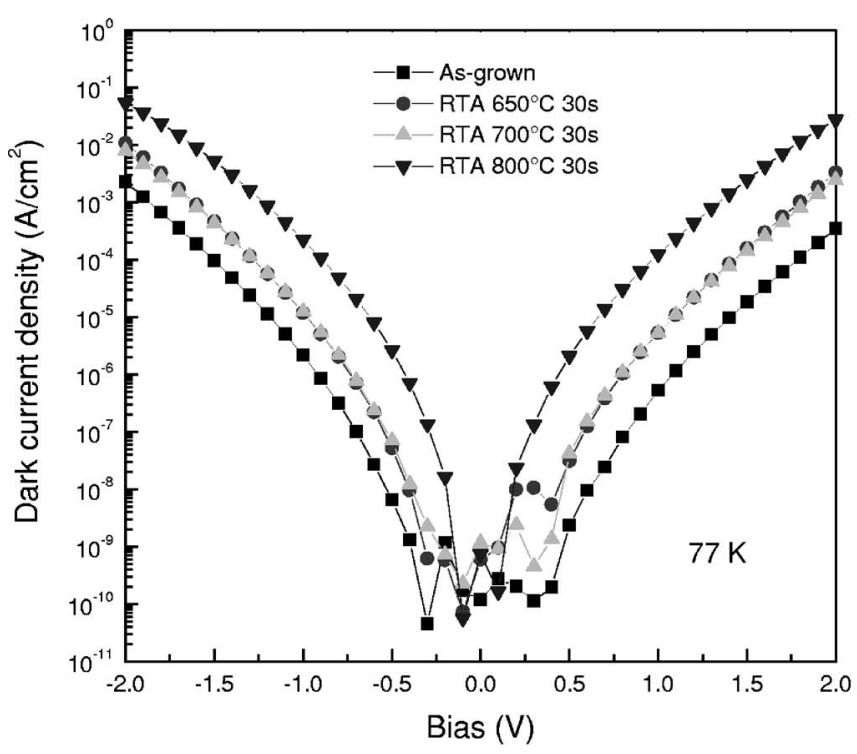

(a)

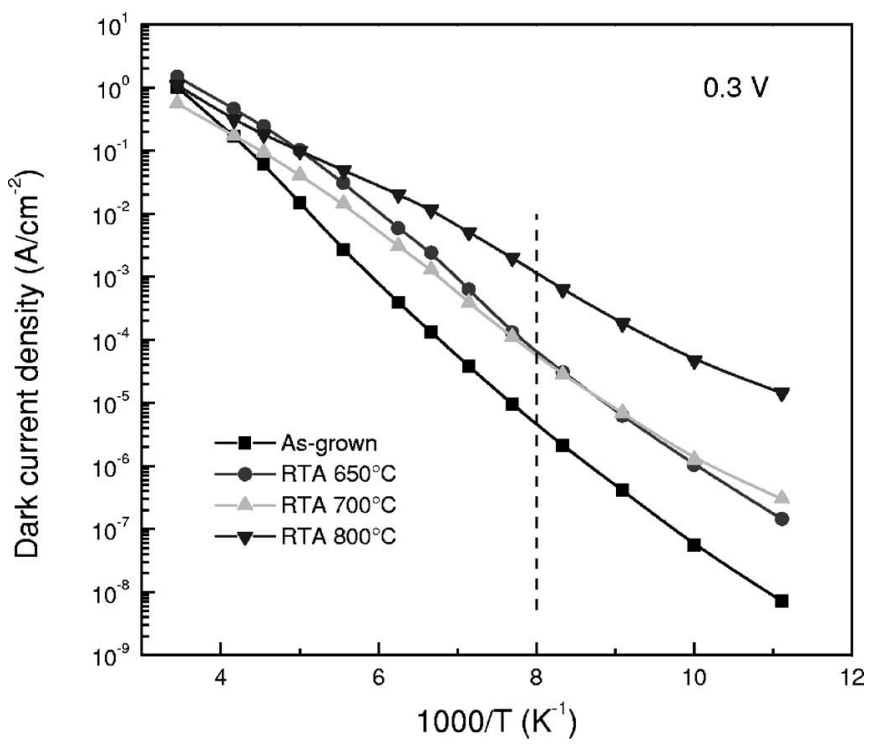

(b)

FIG. 9. (a) The $77 \mathrm{~K} I$ - $V$ characteristics for the annealed QDIPs compared with the as-grown QDIP. (b) The dark current density versus inverse temperature $(1000 / T)$ for the annealed QDIPs compared with the as-grown QDIP at $0.3 \mathrm{~V}$.

temperature curve was increasingly reduced for the 700 and $800{ }^{\circ} \mathrm{C}$ annealed samples due to interdiffusion. However, the similar amount of dark current obtained for both 650 and $700{ }^{\circ} \mathrm{C}$ annealed samples again stresses the important contribution from the defect-related component.

\section{Responsivity and detectivity}

The blackbody response photocurrent $I_{\mathrm{ph}}$ and spectrum of noise current $i_{n}$ for our QDIPs were measured simultaneously using a low-noise current preamplifier (SRS 570) and a fast Fourier transform spectrum analyzer (SRS 760). A germanium block was placed in front of the aperture of the blackbody source to filter out near-IR radiation from the blackbody. ${ }^{3}$ The signal was measured at a modulation frequency of $140 \mathrm{~Hz}$ and the absolute peak responsivity $R_{p}$ was

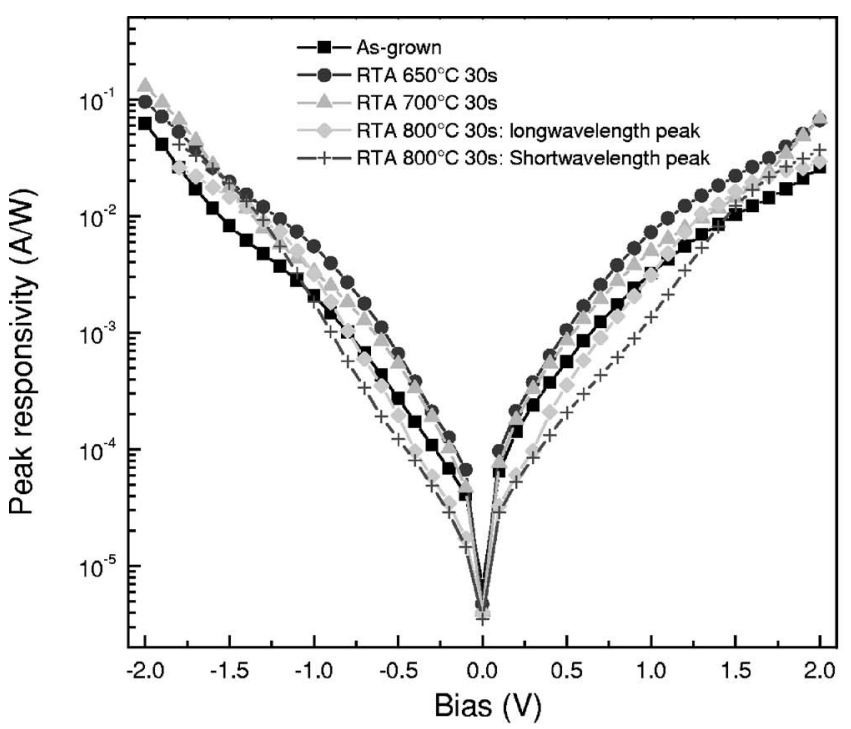

(a)

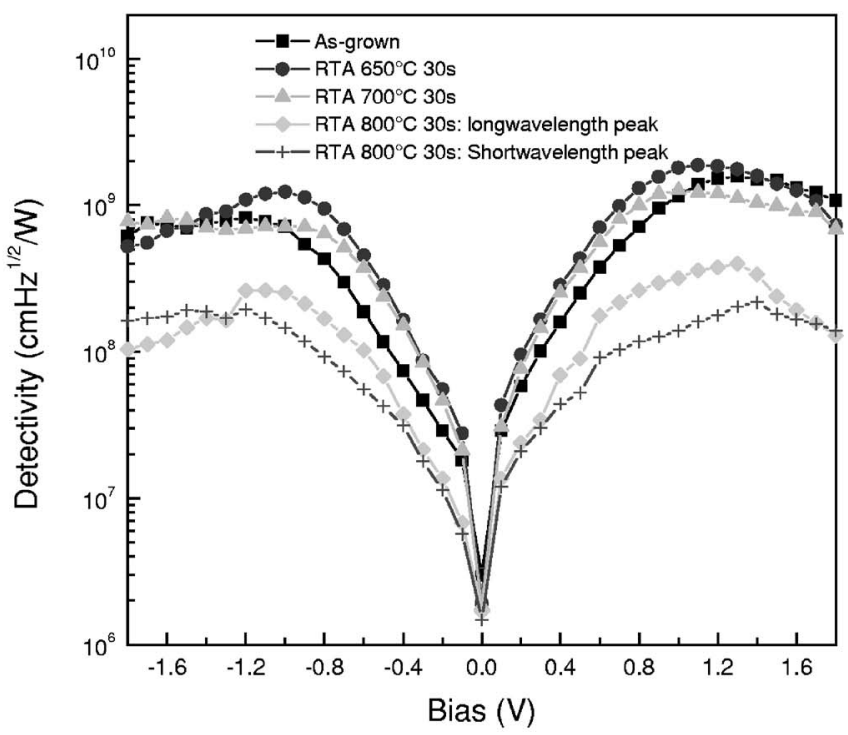

(b)

FIG. 10. (a) The bias dependence of peak responsivity for the annealed QDIPs compared with the as-grown QDIP. (b) The bias dependence of peak detectivity for the annealed QDIPs compared with the as-grown QDIP.

calibrated with an IR-301 blackbody source at $800{ }^{\circ} \mathrm{C}$. Figure 10(a) shows the peak spectral responsivity versus bias for all of the QDIP samples fabricated in this study. For the as-grown QDIP, with increase of positive bias, the responsivity increased from $0.065 \mathrm{~mA} / \mathrm{W}$ at $0.1 \mathrm{~V}$ to $26 \mathrm{~mA} / \mathrm{W}$ at $2 \mathrm{~V}$. For the negative bias, the responsivity is enhanced from $0.041 \mathrm{~mA} / \mathrm{W}$ at $-0.1 \mathrm{~V}$ to $61.8 \mathrm{~mA} / \mathrm{W}$ at $-2 \mathrm{~V}$. The slightly asymmetric shape of the curve between forward and reverse biases could be due to the intrinsically asymmetric nature of QDs. Different from the results in Ref. 16 where the responsivity was largely decreased after thermal treatment, the responsivity of the annealed devices does not show much variation from the as-grown sample, with a marginal improvement at low annealing temperatures. However, the peak responsivity is relatively low and this could be explained by two reasons. Firstly, due to the undoped active region and a large number of QD layers, the supply of car- 
TABLE I. Summary of device properties for all the QDIPs studied in this work.

\begin{tabular}{|c|c|c|c|c|c|}
\hline QDIPs & $\begin{array}{l}\text { Bias } \\
(V)\end{array}$ & $\begin{array}{c}\text { Dark } \\
\text { current } \\
\text { density } \\
\left(\mathrm{A} / \mathrm{cm}^{2}\right)\end{array}$ & $\begin{array}{l}\text { Peak } \\
\text { position } \\
(\mu \mathrm{m})\end{array}$ & $\begin{array}{c}\text { Peak } \\
\text { responsivity }(\mathrm{mA} / \mathrm{W})\end{array}$ & $\begin{array}{c}\text { Peak } \\
\text { detectivity }(\mathrm{cm} \\
\left.\mathrm{Hz}^{1 / 2} / \mathrm{W}\right)\end{array}$ \\
\hline As-grown & 1.3 & $4.98 \times 10^{-6}$ & 6.1 & 6.9 & $1.58 \times 10^{9}$ \\
\hline RTA $650^{\circ} \mathrm{C}$ & 1.1 & $1.1 \times 10^{-5}$ & 6 & 9.6 & $1.89 \times 10^{9}$ \\
\hline RTA $700^{\circ} \mathrm{C}$ & 1 & $5.33 \times 10^{-6}$ & 6.5 & 5 & $1.27 \times 10^{9}$ \\
\hline $\begin{array}{c}\text { RTA } 800{ }^{\circ} \mathrm{C} \\
\text { (long wavelength peak) }\end{array}$ & 1.3 & $7.93 \times 10^{-4}$ & 7.4 & 10.5 & $4 \times 10^{8}$ \\
\hline
\end{tabular}

riers from our QDIP structure through background doping and/or contact layers may not be sufficient to occupy most of the QD states. Secondly, due to the shape of our QDs, which are wide in the in-plane direction $(\sim 20 \mathrm{~nm})$ and narrow in the growth direction $(\sim 5 \mathrm{~nm})$ as a result of the selfassembled growth process, as mentioned earlier, strong confinement has been achieved only in the growth direction. Consequently, the $B-C$ transition that dominates the photocurrent was generated from the z-polarized light. ${ }^{22}$ However, due to the near-normal incidence geometry used in our measurement, only a small amount of $z$-polarized light was produced (from the extrinsic light scattering ${ }^{26}$ ), resulting in the low responsivity in our samples.

The specific peak detectivity was calculated by $D^{*}=R_{p} \sqrt{A_{\operatorname{det}} \Delta f} / i_{n}$, where $A_{\operatorname{det}}$ is the detector area, $i_{n}$ is the noise current, and $\Delta f$ is the bandwidth. Figure 10(b) shows the peak detectivity versus bias curves measured at $77 \mathrm{~K}$. Similar peak detectivities were obtained for the as-grown, and 650 and $700{ }^{\circ} \mathrm{C}$ annealed QDIPs, whereas annealing at $800{ }^{\circ} \mathrm{C}$ caused an obvious reduction of detectivity. Table I summarizes the overall detector performance at $77 \mathrm{~K}$ for all the QDIP samples studied in this work, including the dark current density, the peak detection wavelength, the maximum peak detectivity, as well as the corresponding peak responsivity. It can be noted that the device performances have been well maintained at low annealing temperatures. For the higher annealing temperature of $800^{\circ} \mathrm{C}$, the detector detectivity was reduced; however, the responsivity was not degraded. This could be ascribed to combined effects from increased ground state energy (more photocurrent and dark current) and more defect formation (more dark current). Nevertheless, the degradation of device properties observed in this study is much less in extent compared with other reported studies under similar conditions, ${ }^{16,25}$ which is attributed to the good growth quality of the QDIP structure. By optimizing the structure design, doping, and other parameters of MOCVD growth, the device performance is expected to be further improved and degradation from thermal annealing can also be minimized. These results are encouraging for the future implementation of multicolor detectors using intermixing techniques such as dielectric capping or ion implantation. It has been shown ${ }^{12}$ that in comparison with QWs, large differential wavelength shifts can be achieved in QD structures at low annealing temperatures by low-dose ion implantation or dielectric capping. During these processes only a low density of point defects will be created to induce interdiffusion, which may not cause too much degradation to the device performance. On the other hand, the largely broadened spectrum with two detection peaks obtained by simple annealing at higher temperature is also a desirable feature for broadband imaging applications.

\section{CONCLUSIONS}

The rapid thermal annealing effects on the main device properties of a 15-layer InGaAs/GaAs quantum dot infrared photodetector have been investigated. Compared with the device fabricated from the as-grown structure, low annealing temperatures did not cause much change of the device performance. A higher annealing temperature produced a large wavelength shift (up to $1.3 \mu \mathrm{m}$ ) and although there was some degradation on the detector's peak detectivity, this was relatively small and can be further minimized by improving the QDIP structure design and growth quality. These results suggest that by utilizing intermixing techniques, multicolor or broadband QDIPs can be developed in the future without compromising too much the device performance.

\section{ACKNOWLEDGMENTS}

The authors would like to thank M. Aggett, T. Halstead, and D. Gibson for their continuous technical assistance. The financial support from Australian Research Council is also acknowledged.

${ }^{1}$ Nano-Optoelectronics, Concepts, Physics and Devices, edited by $\mathrm{M}$ Grundmann (Springer, Berlin, 2002)

${ }^{2}$ K. Kamath, P. Bhattacharya, T. Sosnowski, T. Norris, and J. Phillips, Electron. Lett. 32, 1374 (1996).

${ }^{3}$ A. D. Stiff, S. Krishna, P. Bhattacharya, and S. W. Kennerly, IEEE J. Quantum Electron. 37, 1412 (2001)

${ }^{4}$ H. H. Tan and C. Jagadish, Appl. Phys. Lett. 71, 2680 (1997).

${ }^{5}$ L. Fu, H. H. Tan, M. B. Johnston, M. Gal, and C. Jagadish, J. Appl. Phys. 85, 6786 (1999).

${ }^{6}$ M. B. Johnston et al., Appl. Phys. Lett. 75, 923 (1999).

${ }^{7}$ L. Fu, H. H. Tan, C. Jagadish, N. Li, N. Li, X. Q. Liu, W. Lu, and S. C. Shen, Appl. Phys. Lett. 78, 10 (2001).

${ }^{8}$ M. Buda, J. Hay, H. H. Tan, L. Fu, C. Jagadish, P. Reece, and M. Gal, J. Electrochem. Soc. 150, G481 (2003).

${ }^{9}$ R. Leon, Y. Kim, C. Jagadish, M. Gal, J. Zou, and D. J. H. Cockayne, Appl. Phys. Lett. 69, 1888 (1996).

${ }^{10}$ P. Lever, L. Fu, H. H. Tan, C. Jagadish, P. Reece, and M. Gal, in Third IEEE Conference on Nanotechnology, San Francisco, CA, 2003 (IEEE Publishing Co., Piscataway, NJ, 2003), p. 852.

${ }^{11}$ P. Lever, H. H. Tan, C. Jagadish, P. Reece, and M. Gal, Appl. Phys. Lett. 82, 2053 (2003).

${ }^{12}$ L. Fu, P. Lever, H. H. Tan, C. Jagadish, P. Reece, and M. Gal, IEE Proc.: Circuits Devices Syst. 152, 491 (2005)

${ }^{13}$ P. Lever, H. H. Tan, and C. Jagadish, J. Appl. Phys. 96, 7544 (2004).

${ }^{14}$ D. Pan, E. Towe, and S. Kennerly, Appl. Phys. Lett. 73, 1937 (1998) 
${ }^{15}$ S. Krishna, S. Raghavan, G. v. Winckel, P. Rotella, A. Stintz, C. P. Morath, D. Le, and S. W. Kennerly, Appl. Phys. Lett. 82, 2574 (2003).

${ }^{16}$ S. H. Hwang, J. C. Shin, J. D. Song, W. J. Choi, J. I. Lee, and H. Han, Microelectron. J. 36, 203 (2005).

${ }^{17}$ Z. H. Chen, O. Baklenov, E. T. Kim, I. Mukhametzhanov, J. Tie, A. Madhukar, Z. Ye, and J. C. Campbell, J. Appl. Phys. 89, 4558 (2001)

${ }^{18}$ L. Fu, P. Lever, K. Sears, H. H. Tan, and C. Jagadish, IEEE Electron Device Lett. 26, 628 (2005).

${ }^{19}$ L. Fu, P. Lever, H. H. Tan, C. Jagadish, P. Reece, and M. Gal, Appl. Phys. Lett. 82, 2613 (2003).

${ }^{20}$ O. Stier, M. Grundmann, and D. Bimberg, Phys. Rev. B 59, 5688 (1999).

${ }^{21}$ N. Vukmirović, Z. Ikonić, V. D. Jovanović, D. Indjin, and P. Harrison,
IEEE J. Quantum Electron. 41, 1361 (2005).

${ }^{22}$ H. C. Liu, Opto-Electron. Rev. 11, 1 (2003).

${ }^{23}$ Semiconductor Quantum Wells Intermixing, edited by E. H. Li (Gordon and Breach, Amsterdam, 2000).

${ }^{24}$ A. D. Stiff-Roberts, X. H. Su, S. Chakrabarti, and P. Bhattacharya, IEEE Photonics Technol. Lett. 16, 867 (2004).

${ }^{25}$ K. Stewart, M. Buda, J. Wong-Leung, L. Fu, C. Jagadish, A. Stiff-Roberts, and P. Bhattacharya, J. Appl. Phys. 94, 5283 (2003).

${ }^{26}$ H. C. Liu, M. Buchanan, and Z. R. Wasilewski, in International Workshop on Intersubband Transitions in Quantum Wells: Physics and Devices, edited by S. S. Li and Y. K. Su (Kluwer Academic, Taiwan, 1998), p. 50. 